\title{
Screening for Atrial Fibrillation in Zambia's Western Province
}

\author{
K. L. Rush ${ }^{1}$, J. Barker ${ }^{1,2}$, R. Ollivier ${ }^{3}$, M. Ferrier ${ }^{1}$, F. Goma ${ }^{4} \&$ D. Singini ${ }^{5}$ \\ ${ }^{1}$ School of Nursing, University of British Columbia Okanagan, Kelowna, Canada \\ ${ }^{2}$ Interior Health, Kelowna, Canada \\ ${ }^{3}$ School of Nursing, Dalhousie University, Canada \\ ${ }^{4}$ School of Medicine, University of Zambia, Zambia \\ ${ }^{5}$ Limulunga District, Zambian Ministry of Health, Zambia \\ Correspondence: Dr. Kathy Rush, Professor, School of Nursing, University of British Columbia Okanagan, \\ Kelowna, Canada. Tel: 1-250-807-9561. E-mail: Kathy.rush@ubc.ca
}

Received: November 1, 2018 Accepted: December 5, 2018 Online Published: January 7, 2019

doi:10.5539/gjhs.v11n2p1 URL: https://doi.org/10.5539/gjhs.v11n2p1

\begin{abstract}
Background: The prevalence of atrial fibrillation (AF) is increasing in sub-Saharan Africa and contributes to significant morbidity and mortality from stroke and other cardiovascular diseases. Case finding in some areas of the country, such as in rural Zambia is especially challenging with limited availability of ambulatory electrocardiogram monitoring devices. Moreover, there is very little qualitative research exploring Zambian patients' experiences living with AF.
\end{abstract}

Aims: The two-fold purpose of this study was i) to explore the use of a screening tool for detecting AF in Western Province, Zambia and ii) to understand the patient experience of living with AF.

Setting: Mongu and Limulunga Districts in the Western Province of Zambia.

Methods: Mixed methods. Convenience sampling for both quantitative and qualitative arms of the study was used to recruit participants attending public 'Health Checks.' Quantitative data were collected by means of an interview-administered survey and qualitative data were obtained through individual interviews.

Results: An irregular manual pulse was found in $12.6 \%(n=33)$ of Zambians who attended the Health Checks and two participants were confirmed to have AF. Zambians with an irregular pulse had a significantly higher prevalence of hypertension and were significantly less sedentary than those without pulse irregularities. Two themes emerged from the interview with two patients diagnosed with AF: symptoms and healthcare access.

Conclusion: Hypertension continues to be a problem in adult Zambians and is an independent risk factor for stroke and, when diagnosed in conjunction with AF, further compounds stroke risk. Access to screening and diagnostic care for AF is essential, especially in currently underserved rural areas. In addition, participants presented with unique risk factors, such as consolidated periods of exercise, that require further research to determine context and seasonal variation so as to improve education and prevention strategies.

Keywords: atrial fibrillation, cardiovascular disease, hypertension, primary care, screening, sub-Saharan Africa, Zambia

\section{Introduction \& Background}

Atrial fibrillation (AF) in Sub-Saharan Africa (SSA) is on the rise. A 196\% increase in atrial fibrillation was observed from 1990-2014 (Moran et al., 2013) and prevalence is expected to reach approximately 12.5 million in SSA by 2050 (Rahman, Kwan, \& Benjamin, 2014). Incidence of, and death from, cardiovascular disease, including AF, occurs at a younger age than in most developed countries (Lugero, Kibirige, Kayima, Mondo, \& Freers, 2016; Moran et al., 2013; Murray et al., 2015; Ntep-Gweth et al., 2010; Shavida, Yonga, Mwanzi, Jinah, Moriasi, \& Otieno, 2013). There exists a severe lack of data regarding prevalence and incidence of AF in African countries (Zulkifly, Lip, \& Lane, 2018). Of those diagnosed with atrial fibrillation in Africa, $84.1 \%$ have permanent $\mathrm{AF}$, while $9.6 \%$ and $8.9 \%$ have persistent and paroxysmal AF, respectively (Oldgren et al., 2014), increasing their mortality risk. In general, AF increases stroke risk five-fold (Ntep-Gweth et al., 2010) but compared to 46 countries, SSA had the highest prevalence of stroke $(\mathrm{N}=15400)$ (Healey et al., 2016) with lethal 
strokes disproportionately affecting the African population (Roth et al., 2015). Hypertension is considered to be the most common risk factor for AF worldwide (Jardine, Fine, \& Obel, 2014; Lugero et al., 2016; Oldgren et al., 2014), affecting an estimated 10-20 million people in SSA (Opie \& Seedat, 2005), but with 10\% untreated (Healey et al., 2016). Furthermore, patients with AF in African countries have been found to have a high prevalence of rheumatic heart disease (Stambler \& Ngunga, 2015).

Only two studies were found that explored AF in a Zambian setting. Over a 6-month period, Goma \& Kalinchenko (2015) enrolled 36 clinical registry patients ( $42 \%$ male and 58\% female), with a mean age of 45 years (range: 16-90), who presented with AF to the adult medical admission ward of the University Teaching Hospital (UTH) in Lusaka, Zambia. Thirty-nine percent (39\%) were diagnosed with AF on the index admission and $91.7 \%$ had permanent AF. Rheumatic heart disease, hypertensive heart disease, and heart failure were the major risk factors for AF. Treatment of AF was sub-optimal, with few patients receiving rate control or rhythm control medication or oral anticoagulants. Another study completed at UTH found that, of all patients enrolled with CHF, $26.5 \%$ had AF (Jere, Goma, Andrews, Kaluba, \& Kapenda, 2015). The prevalence of AF in CHF was found to be strongly associated with an age of 65 years and above, obesity, smoking, excessive alcohol intake, hypertension, dilated cardiomyopathy, diabetes mellitus, and chronic lung disease.

Little is known about the clinical presentation of AF in Zambian adults who live outside of urban centers. In rural Zambian communities with limited availability of ambulatory monitoring devices such as portable electrocardiograms (ECG's) (Goma \& Kalinchenko, 2015; Rahman et al., 2014), case finding is especially challenging. In a study conducted in rural Tanzania, only $0.67 \%$ of the surveyed population of older adults 70 years of age and older, was found to have AF (Dewhurst et al., 2012), indicating that there may be a 'threshold' in terms of prevalence after a certain age in low-income, rural settings (Gray et al., 2016). Similar findings were reported in a study in rural Ghana, with only $0.3 \%$ of adults 50 years of age and over found to have AF (Koopman, van Bodegom, Westerndorp, \& Wouter Jukema, 2014). IPhone single-lead ECGs that do not require electrode application and are increasingly being used for mass screening for AF have shown promising results for detecting AF when compared to 12-lead ECGs (Lau et al., 2014). Moreover, there is very little qualitative research exploring Zambian patients' experiences of living with AF as a way to enhance understanding of contextual and cultural factors that may influence AF management.

\subsection{Research Aims \& Objectives}

The two-fold purpose of this study was i) to explore the use of a screening tool for detecting AF in Western Province, Zambia and ii) to understand the patient experience of living with AF.

\section{Methods}

\subsection{Design \& Setting}

The mixed methods study took place in Mongu and Limulunga Districts in the Western Province of Zambia. Quantitative and qualitative approaches were used to determine the feasibility of using a screening tool for AF detection complemented by experiential accounts of Zambians prior to and following AF diagnosis. Mixing methods provided a more complete picture of the challenges of Zambians from Western Province in living with and accessing care for AF (Creswell \& Clarke, 2011). Quantitative data were collected by means of an interview-administered survey and qualitative data were obtained through individual interviews.

\subsection{Sampling}

A convenience sample of participants 18 years of age and older were recruited during four public 'Health Checks', two held in Mongu and two in Limulunga Districts. Participation included screening at the Health Check (quantitative arm) and a follow up interview if AF was detected (qualitative arm). 'Health Check' stations were set up in high traffic areas such as the local markets, bus stations, and grocery stores within Mongu and Limulunga towns. Word of mouth and local radio advertisements were used as they had been effective strategies in recruiting sufficient numbers during previous research. A sample size of approximately 200-250 participants was sought, based on approximately 30 participants per variable to provide sufficient power to detect significant relationships (Cohen, Cohen, West, \& Aiken, 2003; VanVoorhis \&Morgan, 2007).

\subsection{Data Collection}

Prior to data collection, informed written consent was obtained. Data collection was completed by nurse educators (JB, FK) and fourth-year nursing students from the University of British Columbia-Okanagan (UBCO), who were in Zambia for a global health practicum during March-April 2017. Health Check sites, each made up of five stations, were managed by a UBCO nursing student and a local Zambian nursing student who was proficient in the 
local language, Silozi. The Zambian nursing students acted as translators and assisted with the consent process. Local health care professionals, such as Clinical Officers, provided onsite medical follow-up as needed.

A modified version of the standardized WHO STEPwise approach to surveillance (World Health Organization, 2010) was used for collection of the following data: blood pressure, body mass index (BMI) (weight, height), waist circumference, and self-reported health behaviours, including alcohol use, smoking, sleep apnea, physical activity (PA), and diet. Blood pressure was measured using the 2014 Clinical Practice Guidelines for the Management of Hypertension in the Community by the American Society of Hypertension and the International Society of Hypertension (Weber et al., 2014). The WHO STEPwise survey methodology recommended three blood pressure measurements separated by at least five minutes, with the average blood pressure calculated from the second and third readings [21]. Hypertension was diagnosed when a person's systolic blood pressure was $\geq 140 \mathrm{~mm} \mathrm{Hg}$ or their diastolic blood pressure was $\geq 90 \mathrm{mmHg}$, or both (Weber et al., 2014; World Health Organization, 2007). Height, weight, and waist circumference were measured using standardized approaches to ensure accuracy and consistency (Goma et al., 2011), with height and weight measurements used to calculate BMI (wt/ht2). PA was assessed using the Global Physical Activity Questionnaire (GPAQ) (Armstrong \& Bull, 2006), a valid and reliable measure of physical activity in urban and rural populations in SSA (Cleland et al., 2014). Sleep apnea was measured using 2 questions: A yes/no question, "Do you wake up at night after going to sleep?" and a frequency question, If YES, how often (once/twice, 3-4 times, 5-6 times, $>6$ times).

At Health Check stations, all participants first had a manual pulse check. A participant found to have an irregular manual heart rate or a history of 'heart palpitations', then had a single lead ECG performed using Kardia Mobile, a cardiac monitoring device attached to an iPhone. Nursing students and health care professionals, trained to use this technology, obtained the ECG by having participants place their left and right fingers on the electrodes, built into the back of the phone. Participants showing an abnormal reading on the Kardia subsequently had a 7-lead ECG, which was set up at another location at the Health Check station. Participants found to have an abnormal Kardia reading were also referred to Lewanika General Hospital (LGH), a provincial referral center, where a physician was assigned to follow them for their suspected AF. At LGH, participants received further diagnostics, including a 12-lead ECG and blood work as ordered by the physician.

For anyone with suspected $\mathrm{AF}$, stroke risk was assessed using the validated $\mathrm{CHA}_{2} \mathrm{DS}_{2}$-VASc score (Olesen, Torp-Pedersen, Hansen, \& Lip, 2012). $\mathrm{CHA}_{2} \mathrm{DS}_{2}$-VASc identifies major risk factors as age $>75$ years and previous stroke/TIA (each allocated two points) and non-major risk factors as congestive heart failure, hypertension, diabetes mellitus, age between 65 years and 75 years, vascular disease, and female biological sex (each allocated one point). The higher the $\mathrm{CHA}_{2} \mathrm{DS}_{2}$-VASc score, the higher the risk of thromboembolism or stroke.

Patients suspected of $\mathrm{AF}$ at the Health Check stations were invited to participate in digitally recorded, semi-structured, individual interviews in order to further understand their experiences with symptoms, as well as issues and challenges with accessing health care, including referrals, treatments, and medications. A translator was available for interviews to enhance participants' comfort with speaking Silozi rather than English. Participants were paid a stipend equivalent to $\$ 10 \mathrm{CAD}$ to cover any expenses (e.g., travel) related to participation in the interview.

\subsection{Data Analysis}

Descriptive statistics were used to summarize demographic data about the sample and study variables. Chi-square tests of association were performed on categorical variables. Independent t-tests were used to compare between group differences (those with, and without, manual pulse irregularity) on hypertension, BMI, WC, physical activity, sleep apnea, smoking, alcohol consumption. All analyses used a $\mathrm{p}<0.05$. Interviews were transcribed (JB) and subsequently coded, with common codes clustered into themes (Richards \& Morse, 2012).

\subsection{Ethical Considerations}

Prior to recruitment and data collection, ethics approval for this research project was obtained from the UBC Okanagan Behavioural Research Ethics Board (H16-03229) and the Biomedical Research Ethics Committee at the University of Zambia (008-01-17), with permission also obtained from the Zambian Ministry of Health.

\section{Results}

Demographics for the study sample $(n=261)$ are reported in Table 1 . Over a quarter of participants were over 60 years of age, approximately two thirds were female, over half were married, and $36.5 \%$ had completed secondary school or above. 
Table 1. Participant demographic information

\begin{tabular}{|c|c|c|c|}
\hline \multicolumn{2}{|c|}{ Demographic characteristics } & \multirow{2}{*}{\multicolumn{2}{|c|}{$\begin{array}{lr}\text { Frequency } & \text { Percent } \\
\text { Range }=18-93 ; \text { Mean }=46.7 ; \mathrm{SD}=17.6\end{array}$}} \\
\hline \multirow{6}{*}{ Age } & Age group & & \\
\hline & $18-29$ & 55 & 21.6 \\
\hline & $30-39$ & 48 & 18.8 \\
\hline & $40-49$ & 42 & 16.5 \\
\hline & $50-59$ & 43 & 16.9 \\
\hline & $60+$ & 67 & 26.2 \\
\hline \multirow{2}{*}{ Sex } & Male & 92 & 35.2 \\
\hline & Female & 169 & 64.8 \\
\hline \multirow{4}{*}{ Marital status } & Never married & 48 & 18.4 \\
\hline & Currently married & 141 & 54.0 \\
\hline & Separated or divorced & 25 & 9.6 \\
\hline & Widowed & 47 & 18.0 \\
\hline \multirow{6}{*}{ Education level } & No formal schooling & 24 & 9.2 \\
\hline & Less than primary school & 51 & 19.5 \\
\hline & Primary school completed & 93 & 35.6 \\
\hline & Secondary school completed & 74 & 28.4 \\
\hline & College/university completed & 17 & 6.5 \\
\hline & Post-graduate degree & 2 & 0.8 \\
\hline \multirow{5}{*}{ Work } & Government employee & 12 & 4.6 \\
\hline & Non-government employee & 18 & 6.9 \\
\hline & Self-employed & 119 & 45.8 \\
\hline & Unemployed (able to work) & 58 & 22.3 \\
\hline & $\begin{array}{l}\text { Other (student, homemaker, retired, unable to } \\
\text { work, non-paid) }\end{array}$ & 53 & 20.4 \\
\hline
\end{tabular}

\subsection{Manual Pulse}

Manual pulse irregularity was found in $12.6 \%$ ( $n=33$ ) of Zambians who attended the Health Checks. More women $(\mathrm{n}=21)$ than men $(\mathrm{n}=12)$ had a manual pulse irregularity but this difference was not significant. Only two Zambians showed abnormal Kardia ECGs, and were confirmed to have AF using a seven-lead ECG. An additional seven participants with detected manual pulse irregularity had seven-lead ECGs used in the field that confirmed other arrhythmias: two with a combination of first and second-degree heart block, four with Premature Ventricular Contractions (PVCs), and one with sinus tachycardia.

Comparison of Zambians with $(n=33)$ and without an $(n=224)$ irregular pulse showed some notable differences (refer to Table 2). Those with an irregular pulse had a significantly higher prevalence of hypertension (51.5\%) compared to those without pulse irregularities $(32.6 \%)$. The mean BP for those with an irregular HR was $136.79 / 80.79 \pm 18.279 / 14.703$ and for those without irregularities was 130.98/78.70 $\pm 19.906 / 11.230$. Surprisingly, those with an arrhythmia spent significantly more days (5.50) participating in vigorous sport and fewer daily sedentary hours (3.56 hours/day) compared to their counterparts with a regular pulse, who spent 3.49 days/week in vigorous sport and 5.39 hours/day in sedentary activity. There was significant difference in self-reported night awakenings $\left(\chi^{2}=9.563, \mathrm{p}=0.023\right)$. Two-thirds $(66.7 \%, \mathrm{n}=22 / 33)$ of those with heart rate irregularities reported waking up once or twice during the night compared to only $52.2 \%(\mathrm{n}=119 / 228)$ of those with a regular heart rate. 
Table 2. Comparison between Participants with Irregular and Regular Manual Pulse

\begin{tabular}{lll}
\hline & Irregular HR $(\mathrm{N}=33)$ & Regular HR $(\mathrm{N}=228)$ \\
\hline Average BMI & 24.9411 & 25.2370 \\
Average waist circumference & 75.9813 & 81.0655 \\
${ }^{a} \mathrm{HTN}$ (Stage I or Stage II) & $51.5 \%(\mathrm{n}=17)$ & $32.0 \%(\mathrm{n}=73)$ \\
Yes to smoking & 4 & 26 \\
Yes to alcohol consumption & 4 & 21 \\
${ }^{\mathrm{b}}$ Average sedentary hours/day & 3.56 & 5.39 \\
Yes to vigorous-intensity sports & 6 & 54 \\
${ }^{\mathrm{c}}$ Average days/week doing vigorous sports & 5.50 & 3.49 \\
Average hours/day doing vigorous sports & 1.75 & 1.90 \\
\hline
\end{tabular}

\footnotetext{
${ }^{\mathrm{a}}$ Significant difference, $\chi 2=4.527$, Significance $=0.033$.

${ }^{\mathrm{b}}$ Significant difference, $\mathrm{t}=2.676$, Significance $=0.010$.

${ }^{\mathrm{c}}$ Significant difference, $\mathrm{t}=2.204$, Significance $=0.031$.
}

\subsection{Patient Experience of Atrial Fibrillation}

Two of the 261 patients screened for AF using the Kardia Mobile, 1-lead ECG were found to have AF; one patient had AF confirmed by a 12 lead ECG at LGH. A 55-year old female with a history of hypertension and taking anti-hypertensive medication, and an 83-year old male with a history of stroke and tuberculosis participated in individual interviews. At the Health Check, the female was experiencing heart palpitations, with a BP 147/96 and a manual pulse $=59$. The male participant was experiencing palpitations, with a $\mathrm{BP}=181 / 81$ and a manual pulse $=68$. $\mathrm{CHA}_{2} \mathrm{DS}_{2}$-VASc score for the female was 2 (low-to-moderate stroke risk) and for the male was 6 (moderate-to-high stroke risk).

Two themes emerged from their interviews: symptoms and healthcare access. Since both patients had been newly identified with AF, their retrospective accounts captured both pre- and post-diagnostic experiences of living with health issues and seeking care/treatment for AF.

\subsubsection{Symptoms}

Both participants described generally feeling unwell in their day to day lives. The female participant described her health as quite poor, stating: "Generally I'm not fine, I feel like I'm very sick." Her description was echoed by the male participant, who stated: "I wouldn't say I'm okay with the way I'm feeling. I can't really say that I'm fine." Common symptoms for both were feelings of a rapidly-beating heart, headache, and chest pain. The female participant described initially experiencing intermittent chest pain that had worsened over time:

But I've been having more pains to my chest and in my back. I've had pricking pain from my chest and across my back, up my neck on the left side. This has all been within the past three months. I have been experiencing it on and off for the past couple of years, but in the last three months it has been coming more often. It just comes with or without exercise.

The male participant described vague intermittent symptoms as "general body weakness, some malaise. Then sometimes my heart would beat fast and then it would stop. Then sometimes I would lose my voice." Other symptoms they described included leg swelling and heaviness, "blood rushing through my ears", and "nighttime throbbing of the heart in my head". Furthermore, both participants expressed feelings of uncertainty related to these symptoms. The female participant described ongoing "issues with stress, because I wonder if my illness is going to kill me."

\subsubsection{Access to Health Care}

Both participants had been to the local primary health centre, staffed by nurses and clinical officers, numerous times during the past year. The female participant had been for monthly visits to the centre and made occasional visits to the hypertension clinic at LGH when finances allowed. The male participant had been to the primary health centre at least six times in the past year, "sometimes for coughing, other times headache, sometimes pains in the chest." However, he had not been seen by any medical professionals beyond the primary health center. 
Both participants expressed dissatisfaction with access to care that primarily focused on two issues: the lack of specialty care and the limited local supply and availability of medications. Compounding their access issues was the challenge of travel costs. Participants' medication issues included the inconsistent availability of medications, the complete lack of some of their prescribed medications, and the use of inappropriate substitution medications in some cases. For example, the female participant, who went monthly to the clinic for medications had experienced being on a diuretic [Moduretic $=$ Lasix $40 \mathrm{mg}$ once daily] that was not in supply during a return clinic visit and she ended up receiving a substitute medication, propranolol [50 $\mathrm{mg}$ once daily], a completely different drug classification. It too had to be discontinued because it made her breathing difficult. Further, she stated of her aspirin prescription, "it has finished, and I don't have any more pills remaining."

The male described a similar experience of going to the pharmacy to have two prescriptions filled - for antiplatelet medications, ASA and clopidogrel - only to be told they were out of stock. He expressed concern about the lack of medication availability, stating "I'm not happy that two of the medications were out of stock. It is frustrating and I want all three medications to be there, so I can take them and get well."

At the time of the Health Check, neither participant had received a referral for AF or been hospitalized in the past year. Neither participant had seen a cardiac specialist but regarded it as an important step in treating their condition. The female participant specifically described the limitations of the primary health centre services and her desire to see a specialist:

"Yes, of course. I come to the clinic and only see the nurses, but they are not specialists. I come to the clinic and they say it is my BP, but they do not consider what causes the BP to be high. That's why I want to be seeing a specialist here."

Following the identification of AF using iPhone ECG, both participants were referred to LGH for further follow-up. The female participant couldn't afford the travel required for follow-up and died from unknown causes in Spring 2018. Despite enormous financial hardship, the male participant was able to make the trip to LGH, where he received physician care. A 12-lead ECG confirmed atrial fibrillation and an echocardiogram confirmed left ventricular hypertrophy and congestive heart failure. He continued to demonstrate severe, uncontrolled hypertension (BP: 180/110).

\section{Discussion}

Two cases of AF were detected using a combination of manual pulse palpation and a handheld ECG device during Health Checks in Western Province. These findings are similar to a prevalence study of AF conducted in a rural and remote region of Ghana, in which AF was detected in only 3 cases (Koopman et al., 2014). Unlike the current study, in which ECG evaluation was performed only with prior assessment of an irregular manual pulse, all patients were assessed using ECGs (12-leads) only. In the current study, detected cases of AF may have been under-represented as only those Health Check participants who had an irregular palpated pulse had one-lead ECGs performed. Further, palpation and not auscultation of heart rate may have failed to detect arrhythmias. Student nurses who were collecting the data were trained and had experience in pulse palpation but overall inexperience may have contributed to their missing irregularities and potential AF cases. Owing to a limited supply of Kardia devices, it was practically difficult to obtain one-lead ECGs on all participants. The small sample size for the survey $(\mathrm{n}=261)$ and the possibility of participant self-selection limit representativeness and generalizability of findings with future research needed involving a larger sample from a broader geographical area in Western Province.

Hypertension continues to be a problem in adult Zambians and is an independent risk factor for stroke and, when diagnosed in conjunction with $\mathrm{AF}$, further compounds stroke risk. Both patients who were diagnosed with $\mathrm{AF}$ had hypertension; however, medication availability for hypertension was very problematic for them. The lack or inconsistent availability of medications has been reported by other Zambian researchers. Goma and Kalinchenko (2015) found that 12 of 36 (33.3\%) patients with AF in their study had a history of hypertension but only 9 (75\%) of them were on medication. Based on their $\mathrm{CHA}_{2} \mathrm{DS}_{2}$-VASc, both patients with $\mathrm{AF}$ in the current study were at risk of stroke and candidates for anticoagulants or platelet inhibitors (e.g., clopidogrel), though the medications were largely unavailable. Adding to the lack of appropriate treatment was the unavailability of certain diagnostic tests, such as International Normalized Ratio (INR) blood testing. This lack of laboratory capacity to determine INR has been identified as a main barrier to the use of warfarin; without such monitoring, it is considered risky to begin anticoagulation therapy (Goma \& Kalinchenko, 2015).

Obesity, smoking, and obstructive sleep apnea (OSA) are known risk factors for AF. Overall, Zambian participants were found to be marginally overweight, a finding similar to a study of urban Zambian patients with AF (Goma \& 
Kalinchenko, 2015). In the current study, only self-reported sleep patterns were found to be significantly different between those with, and without, pulse irregularity. While it is not known whether these awakenings were due to OSA, it is the most prevalent sleep disorder in Africa and Africans have shown a genetic predisposition to OSA (Aragon-Arreola, Moreno-Villegas, Armienta-Rojas, \& De la Herran-Arita, 2016).

Participants in the current study, many from impoverished and rural communities such as Mongu and Limulunga Districts, rely on local health centres as their primary care access. However, both participants with confirmed AF were dissatisfied with the limitations in accessibility and type of care at their local facilities. Further study of the patient experience with care seeking and especially follow-up would contribute valuable information for enhancing services. Staff at local health facilities often lack both an understanding of chronic conditions such as $\mathrm{AF}$ and hypertension and training in their prevention, screening, and management. In the environments, typical to Mongu and Limulunga Districts, health care workers also face diagnostic challenges (e.g., thromboembolism, vascular and CHF history). As such, it is imperative they have the tools and training necessary to work to their full scope and provide basic care/treatment services for NCDs, including AF, with referrals to major centres as necessary. Research aimed at exploring the perspectives of Zambian HCPs related to current screening practices and viable approaches for promoting screening would provide much needed contextual knowledge to begin capacity-building to address current gaps in healthcare. The prevention and management of AF, currently under-emphasized in SSA, needs to be made a national priority with regards to health policies and allocated resources.

It is worth noting that those participants with arrhythmias were more active and less sedentary on a daily basis compared to those with a regular manual pulse. Although not specific to AF, this finding is consistent with the growing evidence showing that endurance athletes are more likely to develop atrial fibrillation (AF) than non-athletes (Flannery, Kalman, Sanders, \& La Gerche, 2017). This evidence perhaps points to a need to promote regular, moderate exercise rather than intense periods of physical work or activity. Given the lifestyle of rural participants there may be considerable seasonal variation in level of activity, as many participants in the study areas make the majority of their livelihood as farmers. While it was found that participants were generally active several days per week, the period of activity per day was quite consolidated. Participants were either sedentary or performing vigorous activity but there were few reports of daily integrated activity, such as cycling or walking.

\subsection{Implications}

This study has implications for arrhythmia screening in populations in SSA with more limited access to services for $\mathrm{AF}$ detection. Use of manual pulse alone was able to detect a $12.6 \%$ prevalence of arrhythmia in the Zambia study population. Although AF was detected in only 2 patients, $27.3 \%$ of all patients who had an irregular manual pulse had heart rhythm disturbances confirmed by ECG (7 and 12-lead). Manual pulse palpation has been criticized for its low specificity (Ombonie \& Verberk, 2016), compared to BP monitors and non-12 lead ECGs (Taggar, Coleman, Lewis, Heneghan, Jones, 2016), yet it has been shown to be reliable in selective populations (Jaakkola, Vasankari, Virtanen, Airaksinen, 2017). In Western Province, where equipment is a rare commodity, manual pulse palpation can be used as a regular screening tool. Manual pulse does not negate the need for equipment such as a confirmatory 12-lead ECG but is an important first step towards early detection. With only one ECG machine in the whole of Western Province, there is an urgent need to advocate both for simple mobile devices for AF screening/detection and 12-lead ECG equipment for confirming AF cases.

Equally important to equipment is training in the interpretation of ECGs. Currently, there is only one physician in the province who has the knowledge required to interpret ECGs. Specific training in ECG interpretation could be provided for health care workers, such as Clinical Officers or Medical Officers, as this skill can be easily taught in short-term theory workshops or courses held throughout various health districts. In some health care facilities in SSA, nurses perform ECGs and this is a skill Zambian nurses could also be taught. Building capacity in ECG reading and interpretation would enhance opportunities for AF screening and diagnosis, particularly for patients living with hypertension and others who are otherwise identified as being at risk for developing AF. This could potentially decrease the pre-diagnostic gap for patients living with AF in SSA. Although the 55-year old female participant died only one year after being diagnosed with AF, albeit of unknown cause(s), her story might have been different with earlier detection at the health centre, where she often received care. Determining stroke risk for patients with $\mathrm{AF}$ is imperative, yet in Western Province, diagnostic challenges (e.g. vascular, thromboembolism, $\mathrm{CHF}$ ) make it particularly difficult to accurately ascertain stroke risk using $\mathrm{CHA}_{2} \mathrm{DS}_{2}$-VASc. Additionally, the $\mathrm{CHA}_{2} \mathrm{DS}_{2}$-VASc does not account for race. Kabra, Girotra, \& Vaughan Sarrazin (2016) found that adding race to the $\mathrm{CHA}_{2} \mathrm{DS}_{2}$-VASc improved stroke prediction for African-American Medicare recipients with AF.

Accessibility to screening plays an exceptionally important role in facilitating the diagnosis of NCDs in 
low-income countries, especially in rural areas where facilities or trained health staff may be limited. Examples such as the Kardia technology provide a way to facilitate easy, quick, and affordable screening while also being very portable. However, it remains important for the Zambian Ministry of Health to provide regular opportunities for rural residents to access similar 'Health Checks' for such screening. Participants were keen to attend the facilitated Health Checks, demonstrating a need for mobile care centres/clinics in many areas in Western Province.

\section{Conclusion}

This study builds upon the knowledge required to assess health service and human resources for health needs in relation to cardiovascular disease in Western Province, Zambia. It is clear that more resources are required, though our results also point to a rather simple and affordable solution through use of the Kardia screening tool. With the current limited access or availability of community health screening services, such as those performed in this study, there exists an opportunity to mobilize health care professionals in both the acute care and public health sectors to better meet the needs of the population in Western Province. The literature is clear; non-communicable diseases are a major issue in SSA and are becoming increasingly visible in terms of morbidity and mortality. Upstream approaches, such as screening and subsequent referral, may be utilized to promote positive health outcomes for patients living with atrial fibrillation. Diagnosis is an essential first step to treatment and no less important is follow-up and monitoring of patients diagnosed with $\mathrm{AF}$ as reflected in the experiences of two patients diagnosed with AF. Consistent, reliable access to skilled health services allows for challenges or barriers to care, such as unavailability of medications, to be addressed while also assisting patients with navigating the health care system. Overall, atrial fibrillation is a growing issue and health services must adjust to prioritize the prevention, education, and screening of such chronic diseases, which are complex and interconnected.

\section{Acknowledgements}

The authors have no competing interests to declare. All authors meet the requirements of authorship. A Faculty of Health and Social Development Internal Health Research Grant (PG\#: 62R75088), from the University of British Columbia, Okanagan supported this research. We would like to acknowledge the contributions of Fay Karp, as well as the University of British Columbia Okanagan and Lewanika College of Nursing students for their invaluable assistance with data collection. A special thanks to all those who participated in the health checks and made this study possible. The views expressed in this article are the authors' own and not an official position of the institution or funder.

\section{Authors' Contributions}

Kathy Lynn Rush, Fastone Matthew Goma, Jessica Amelia Barker, Rachel Ann Ollivier, and Douglas Singini conceived and designed the study and were involved in obtaining ethic approvals from their respective organizations. Jessica Amelia Barker and Douglas Singini collected the data. Kathy Lynn Rush and Matthew Scott Ferrier analyzed and interpreted the results. Kathy Lynn Rush and Rachel Ann Ollivier drafted the initial manuscript. All authors reviewed and approved the final manuscript.

\section{Disclaimer}

The views expressed in this article are the authors' own and not an official position of the institution or funder.

\section{Support}

A Faculty of Health and Social Development Internal Health Research Grant (PG\#: 62R75088), from the University of British Columbia, Okanagan supported this research.

\section{Competing Interest Statement}

The authors have no conflicts of interest to declare.

\section{References}

Aragón-Arreola, J. F., Moreno-Villegas, C. A., Armienta-Rojas, D. A., \& De la Herrán-Arita, A. K. (2016). An insight of sleep disorders in Africa. eNeurologicalSci, 3, 37-40. https://doi.org/10.1016/j.ensci.2016.02.006

Armstrong, T., \& Bull, F. (2006). Development of the World Health Organization Global Physical Activity Questionnaire (GPAQ). Journal of Public Health, 14, 66-70. https://doi.org/10.1007/s10389-006-0024-x

Cleland, C. L., Hunter, R. F., Kee, F., Cupples, M. E., Sallis, J. F., \& Tully, M. A. (2014). Validity of the Global Physical Activity Questionnaire (GPAQ) in assessing levels and change in moderate-vigorous physical activity and sedentary behaviour. BMC Public Health, 14, 1255. https://doi.org/10.1186/1471-2458-14-1255

Cohen, J., Cohen, P., West, S. G., \& Aiken, L. A. (2003). Applied multiple regression/correlation analysis for the 
behavioral sciences (3rd ed.). Mahwah NJ: Lawrence Erlbaum Associates Publishers.

Creswell, J. W., \& Clark, V. L. P. (2011). Designing and conducting mixed methods research. Thousand Oaks: Sage Publications.

Dewhurst, M. J., Adams, P. C., Gray, W. K., Dewhurst, F., Orega, G. P., Chaote, P., \& Walker, R. W. (2012). Strikingly low prevalence of atrial fibrillation in elderly Tanzanians. Journal of the American Geriatric Society, 60(6), 1135-1140. https://doi.org/10.1111/j.1532-5415.2012.03963.x

Flannery, M. D., Kalman, J. M., Sanders, P., \& La Gerche, A. (2017). State of the Art Review: Atrial Fibrillation in Athletes. Heart, Lung and Circulation, 26(9), 983-989. https://doi.org/10.1016/j.hlc.2017.05.

Goma, F. M., Nzala, S. H., Babaniyi, O., Songolo, P., Zyaambo, C., Rudatsikira, E., ... \& Muula, A. S. (2011). Prevalence of hypertension and its correlates in Lusaka urban district of Zambia: a population based survey. International Archives of Medicine, 4(1), 34. https://doi.org/10.1186/1755-7682-4-34

Goma, F. M., \& Kalinchenko, S. (2015). Atrial fibrillation in Lusaka: Pathoaetiology, pathophysiology and clinical management challenges in primary care settings. Medical Journal of Zambia, 42(1), 31-41.

Gray, W. K., Dewhurst, F., Dewhurst, M. J., Orega, G., Kissima, J., Chaote, P., \& Walker, R.W. (2016). Rates and predictors of three-year mortality in older people in rural Tanzania. Archives of Gerontology and Geriatrics, 62, 36-42. https://doi.org/10.1016/j.archger.2015.10.008

Healey, J. S., Oldgren, J., Ezekowitz, M., Zhu, J., Pais, P., Wang, J., ... \& Damasceno, A. (2016). Occurrence of death and stroke in patients in 47 countries 1 year after presenting with atrial fibrillation: a cohort study. The Lancet, 388(10050), 1161-1169. https://doi.org/10.1016/S0140-6736(16)30968-0

Jaakkola, J., Vasankari, T., Virtanen, R., \& Airaksinen, K. E. J. (2017). Reliability of pulse palpation in the detection of atrial fibrillation in an elderly population. Scandinavian Journal of Primary Health Care, 35(3), 293-298. https://doi.org/10.1080/02813432.2017.1358858

Jardine, R. M., Fine, J., \& Obel, I. W. P. (2014). A survey on the treatment of atrial fibrillation in South Africa. The South African Medical Journal, 104(9), 623-627. https://doi.org/10.7196/SAMJ.8111

Jere, M., Goma, F. M., Andrews, B., Kaluba, L., \& Kapenda, C. (2015). Clinical Factors Associated with Atrial Fibrillation in Congestive Heart Failure Patients Admitted to the University Teaching Hospital, Lusaka, Zambia. Cardiology and Angiology: An International Journal, 4, 3. https://doi.org/10.9734/CA/2015/19731

Kabra, R., Girotra, S., \& Vaughan Sarrazin, M. (2016). Refining stroke prediction in atrial fibrillation patients by addition of African-American ethnicity to CHA2DS2-VASc score. Journal of the American College of Cardiology, 68(5), 461-470. https://doi.org/10.1016/j.jacc.2016.05.044

Koopman, J. J. E., van Bodegom, D., Westendorp, R. G. J., \& Wouter Jukema, J. (2014). Scarcity of atrial fibrillation in a traditional African population: a community-based study. BMC Cardiovascular Disorders, 14, 87. https://doi.org/10.1186/1471-2261-14-87

Lau, J. K., Lowres, N., Neubeck, L., Brieger, D. B., Sy, R. W., Galloway, C. D., Albert, D. E., \& Freedman, S. B. (2013). iPhone ECG application for community screening to detect silent atrial fibrillation: A novel technology to prevent stroke. International Journal of Cardiology, 165(1), 193-212. https://doi.org/10.1016/j.jicard.2013.01.220

Lugero, C., Kibirige, D., Kayima, J., Mondo, C. K., \& Freers, J. (2016). Atrial fibrillation among the black population in a Ugandan tertiary hospital. International Journal of General Medicine, 9, 191-198. https://doi.org/10.2147/IJGM.S100637

Moran, A., Forouzanfar, M., Sampson, U., Chugh, S., Feigin, V., \& Mensah, G. (2013). The epidemiology of cardiovascular diseases in sub-saharan Africa: The global burden of diseases, injuries and risk factors 2010 study. Progress in Cardiovascular Diseases, 56(3), 234-239. https://doi.org/10.1016/j.pcad.2013.09.019

Mensah, G. A., Sampson, U. K., Roth, G. A., Forouzanfar, M. H., Naghavi, M., Murray, C. J., ... \& Feigin, V. L. (2015). Mortality from cardiovascular diseases in sub-Saharan Africa, 1990-2013: a systematic analysis of data from the Global Burden of Disease Study 2013. Cardiovascular journal of Africa, 26(2 H3Africa Suppl), S6-S10. https://doi.org/10.5830/CVJA-2015-036

Ntep-Gweth, M., Zimmermann, M., Meiltz, A., Kingue, S., Ndobo, P., Urban, P., \& Bloch, A. (2010). Atrial fibrillation in Africa: Clinical characteristics, prognosis, and adherence to guidelines in Cameroon. EP: Europace, 12(4), 482-487. https://doi.org/10.1093/europace/euq006 
Oldgren, J., Healey, J.S., Ezekowitz, M., Commerford, P., Avezum, A., Pais, P., ... \& Yusuf, S. (2014). Variations in cause and management of atrial fibrillation in a prospective registry of 15400 emergency department patients in 46 countries: The RE-LY atrial fibrillation registry. Circulation, 129(15), 1568-1576. https://doi.org/10.1161/CIRCULATIONAHA.113.005451

Olesen, J. B., Torp-Pedersen, C., Hansen, M. L., \& Lip, G. Y. (2012). The value of the CHA2DS2-VASc score for refining stroke risk stratification in patients with atrial fibrillation with a CHADS2 score 0-1: a nationwide cohort study. Thrombosis and Haemostasis: Internal Medicine, 107(6), 1172-9. https://doi.org/10.1160/TH12-03-0175

Ombonie, S., \& Verberk, W. J. (2016). Opportunistic screening of atrial fibrillation by automatic blood pressure measurement in the community. BMJ Open, 6(4), e 010745. https://doi.org/10.1136/bmjopen-2015-010745

Opie, L. H., \& Seedat, Y. K. (2005). Hypertension in sub-Saharan African populations. Circulation, 112(23), 3562-3568. https://doi.org/10.1161/CIRCULATIONAHA.105.539569

Rahman, F., Kwan, G. F., \& Benjamin, E. J. (2014). Global epidemiology of atrial fibrillation. Nature Reviews Cardiology, 11(11), 639-654. https://doi.org/10.1038/nrcardio.2014.118

Richards, L., \& Morse, J. M. (2012). Readme First for User's Guide to Qualitative Methods (3rd ed.). Thousand Oaks: Sage Publications.

Roth, G. A., Huffman, M. D., Moran, A. E., Feigin, V., Mensah, G. A., Naghavi, M., \& Murray, C. L. (2015). Global and regional patterns in cardiovascular mortality from 1990 to 2013. Circulation, 132(17), 1667-1678. https://doi.org/10.1161/CIRCULATIONAHA.114.008720

Shavadia, J., Yonga, G., Mwanzi, S., Jinah, A., Moriasi, A., \& Otieno, H. (2013). Clinical characteristics and outcomes of atrial fibrillation and flutter at the Aga Khan university hospital, Nairobi. Cardiovascular Journal of Africa, 24(2), 6-9. https://doi.org/10.5830/CVJA-2012-064

Stambler, B. S., \& Ngunga, L. M. (2015). Atrial fibrillation in sub-saharan Africa: Epidemiology, unmet needs, and treatment options. International Journal of General Medicine, 8, 231-242. https://doi.org/10.2147/IJGM.S84537

Taggar, J. S., Coleman, T., Lewis, S., Heneghan, C., \& Jones, M. (2016). Accuracy of methods for detecting an irregular pulse and suspected atrial fibrillation: A systematic review and meta-analysis. European Journal of Preventive Cardiology, 23(12), 1330-1338. https://doi.org/10.1177/2047487315611347

VanVoorhis, C. R. W., \& Morgan, B. L. (2007). Understanding Power and Rules of Thumb for Determining Sample Sizes. Tutorials in Quantitative Methods for Psychology, 3(2), 43-50. https://doi.org/10.20982/tqmp.03.2.p043

Weber, M. A., Schiffrin, E. L., White, W. B., Mann, S., Lindholm, L. H., Kenerson, J. G., ... \& Cohen, D. L. (2014). Clinical practice guidelines for the management of hypertension in the community: a statement by the American Society of Hypertension and the International Society of Hypertension. The journal of clinical hypertension, 16(1), 14-26. https://doi.org/10.1111/jch.12237

World Health Organization [WHO]. (2007). Prevention of Cardiovascular Disease Guidelines for assessment and management of cardiovascular risk. Retrieved December 13, 2016 from http://ish-world.com/downloads/activities/71665_71665_OMS_INT-RETIRATION.pdf

World Health Organization [WHO]. (2010). The WHO STEPwise approach to chronic disease risk factor

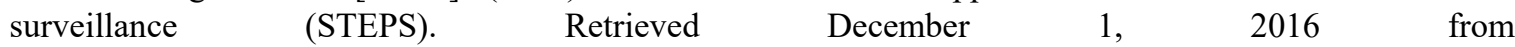
http://www.who.int/chp/steps/manual/en/index.html

Zulkifly, H., Lip, G. Y. H., \& Lane, D. A. (2018). Epidemiology of atrial fibrillation. International Journal of Clinical Practice, 72(3), 1. https://doi.org/10.1111/ijcp.13070

\section{Copyrights}

Copyright for this article is retained by the author(s), with first publication rights granted to the journal.

This is an open-access article distributed under the terms and conditions of the Creative Commons Attribution license (http://creativecommons.org/licenses/by/4.0/). 